УДК 519.873

\title{
Nonlinear Methods of Statistical Analysis of Dynamics of the Tracking Systems in Radio Receivers
}

\author{
Vladimir A. Mironov* \\ Military educational scientific center air force «VVA» \\ Bolshevikov, 54a, Voronezh, 394064 \\ Russia \\ Dmitriy D. Dmitriev ${ }^{\dagger}$ \\ Valeriy N. Tyapkin ${ }^{\ddagger}$ \\ Aleksey Yu. Ershov ${ }^{\S}$ \\ Military Engineering Institute \\ Siberian Federal University \\ Svobodny, 79, Krasnoyarsk, 660041
}

Russia

Received 20.03.2018, received in revised form 26.03.2018, accepted 10.07.2018

The article assesses the criteria ratios (required protection ratios) of the signal / noise that ensure the functioning of the radio receiving device in conditions of a noise immunity deficit with a probability of not less than 0.9. The assessment has been performed using Markov's chain (processes) on the basis nonlinear methods for analyzing the statistical dynamics of tracking systems and the theory of optimal filtering of data processes.

Keywords: Adaptive radio receivers, noise immunity, optimal algorithm, synthesis, tracking. DOI: 10.17516/1997-1397-2018-11-5-627-633.

\section{Introduction}

Modern ground-based command and measuring systems for managing the flight of spacecraft, besides their standard task of receiving command and program data and telemetry, are often required to measure navigational parameters (pseudodistance and pseudospeed) via a received useful signal for the purpose of ephemeris provision [1]. In order to solve this task it is necessary to construct a radio receiver capable of tracking the parameters of the received signal with a high degree of accuracy. Measurable parameters for navigational support include time delay and phase. Both parameters are tracked using delay tracking systems (DTS) and phased automatic frequency adaptors (PLL). The measurement accuracy of these parameters and the possibility of tracking them strongly depend on the noise immunity of the radio receiver.

\footnotetext{
*mirvam@live.ru

†dmitriev121074@mail.ru

†tyapkin58@mail.ru

§alexeyworking@mail.ru

(c) Siberian Federal University. All rights reserved
} 


\section{Estimate of required protection ratio signal / noise}

In this section we shall discuss the procedure for evaluating the immunity ratio of signal / noise which enables radio receivers to operate in conditions of a deficit of interference resistance with a probability of less than 0.9 . The time and probability characteristics of the failure of the signal tracking process for a radio receiver it determined using nonlinear methods for analyzing the statistical dynamics of the tracking systems; these methods are based on the Markov chain $[2,3,4]$. The application of these methods allows us to determine the algorithm for calculating the average time and time dispersion to the tracking failure simultaneously for both PLL and DTS considering coupling. Simultaneous signal tracking failure in DTS and PLL is understood as the first exit of the trajectory of the phase $\varphi$ and delay $\tau_{d}$ beyond the limits of the discrimination characteristics. According to $[4,5,6]$ joint statistical dynamics for DTS and PLL can be viewed in the coordinates

$$
\varphi=\hat{\varphi}-\varphi, \quad \tau_{d}=\hat{\tau}-\tau,
$$

where $\varphi$ is the phase difference, $\tau_{d}$ is the difference of the time position of the base and input signals, $\hat{\varphi}, \hat{\tau}$ are their values.

The characteristics of tracking failure were determined using the following differential equations:

$$
\begin{aligned}
\frac{d \varphi}{d t} & =-\alpha \varphi-\Delta \varphi\left[\sin 2 \varphi+\frac{4 \cos \varphi}{A T_{0}\left(1-\frac{\left|\tau_{d}\right|}{\tau_{e}}\right)} n_{1}(t)+\right. \\
& \left.+\frac{4 \sin \varphi}{A T_{0}\left(1-\frac{\left|\tau_{d}\right|}{\tau_{e}}\right)} n_{2}(t)+\frac{8}{A^{2} T_{0}^{2}\left(1-\frac{\left|\tau_{d}\right|}{\tau_{e}}\right)^{2}} n_{1}(t) n_{2}(t)\right]-n_{3}(t), \\
\frac{d \tau_{d}}{d t} & =-\beta \tau-\Delta \tau\left[F\left(\tau_{d}\right)+\frac{2 F\left(\tau_{d}\right)}{A T_{0}\left(1-\frac{\left|\tau_{d}\right|}{\tau_{e}}\right) \cos \varphi} n_{2}(t)+\right. \\
& \left.+\frac{2 \tau_{e}}{A T_{0} \cos \varphi} n_{3}(t)+\frac{4 \tau_{e}}{A^{2} T_{0}^{2}\left(1-\frac{\left|\tau_{d}\right|}{\tau_{e}}\right) \cos ^{2} \varphi} n_{2}(t) n_{3}(t)\right]-n \tau(t),
\end{aligned}
$$

where $N_{0}$ is the power spectral density of interference; A is signal amplitude; $\Delta \varphi$ is the synchronization (locking) band of the PLL system; $\Delta \tau$ is the locking band of the DTS; $\tau_{e}, T_{0}$ is the duration of the impulse of the pseudorandom sequence and, accordingly, the duration of the data symbol.

$$
\begin{gathered}
\Delta \varphi=\frac{A^{4} T_{0}\left(1-{\frac{\left|\tau_{d}\right|}{\tau_{e}}}^{2}\right)}{2 N_{0}^{2}} \bar{k}_{11} ; \quad \Delta \tau=\frac{A^{4} T_{0} \cos ^{2} \varphi\left(1-\frac{\left|\tau_{d}\right|}{\tau_{e}}\right)}{2 N_{0}^{2} \tau_{e}} \bar{k}_{22}, \\
n_{1}(t)=\int_{(k-1) \cdot T_{0}}^{k \cdot T_{0}} n_{p}(t) g(t-\hat{\tau}) \sin \hat{\Phi} d t, \quad n_{2}(t)=\int_{(k-1) \cdot T_{0}}^{k \cdot T_{0}} n_{d}(t) g(t-\hat{\tau}) \cos \hat{\Phi} d t, \\
n_{3}(t)=\int_{(k-1) \cdot T_{0}}^{k \cdot T_{0}} n_{d}(t) \frac{d g(t-\hat{\tau})}{d \hat{\tau}} \cos \hat{\Phi} d t
\end{gathered}
$$

- noise, which is a normal random process;

$\mathrm{g}(\mathrm{t})$ is the pseudorandom sequence of impulses; 
$F\left(\tau_{p}\right)$ is a function which characterizes the discriminate feature of the DTS; it has the following view

$F\left(\tau_{d}\right)=\frac{\tau_{e}}{T_{0}} \int_{(k-1) \cdot T_{0}}^{k \cdot T_{0}} g(t-\tau) \frac{d g(t-\hat{\tau}) d t}{d \hat{\tau}}=\frac{\tau_{e}}{T_{0}} \int_{(k-1) \cdot T_{0}}^{k \cdot T_{0}} g(t-\tau) \frac{g\left(t-\hat{\tau}+\frac{1}{2 \tau_{e}}\right)-g\left(t-\hat{\tau}-\frac{1}{2 \tau_{e}}\right)}{\tau_{e}} d t$.

After a piecewise linear approximation, the function $F\left(\tau_{d}\right)$ can be viewed as such

$$
F\left(\tau_{d}\right)=\left\{\begin{array}{ccc}
1-\frac{\left|\tau_{d}+2 \tau_{e}\right|}{\tau_{d}} & \text { provided } & -\frac{3}{2} \tau_{e}<\tau_{d}<-\frac{1}{2} \tau_{e}, \\
\frac{\left|\tau_{d}-\frac{1}{2} \tau_{e}\right|}{\tau_{e}}-\frac{\left|\tau_{d}+\frac{1}{2} \tau_{e}\right|}{\tau_{e}} & \text { provided } & -\frac{1}{2} \tau_{\mathrm{e}}<\tau_{p}<-\frac{1}{2} \tau_{\mathrm{e}} \\
\frac{\left|\tau_{p}-\frac{1}{2} \tau\right|}{\tau}-1 & \text { provided } & -\frac{1}{2} \tau_{\mathrm{e}}<\tau_{p}<-\frac{3}{2} \tau_{\mathrm{e}} \\
0 & \text { provided } & -\frac{3}{2} \tau_{\mathrm{e}}>\tau_{p}>\frac{3}{2} \tau_{\mathrm{e}} .
\end{array}\right.
$$

For equation (1) we have considered the product of the accepted oscillation $\xi_{1}(t)$ and the base signal can be shown as:

$$
\begin{aligned}
& \xi_{1}(t) g(t-\hat{\tau}) \cos \left(\omega_{0} t+\hat{\psi}\right)=\frac{1}{2} A\left(1-\frac{\left|\tau_{d}\right|}{\tau_{e}} \cos \varphi\right)+n_{d}(t) g(t-\hat{\tau}) \cos \left(\omega_{0} t+\hat{\psi}\right) \\
& \xi_{1}(t) g(t-\hat{\tau}) \sin \left(\omega_{0} t+\hat{\psi}\right)=\frac{1}{2} A\left(1-\frac{\left|\tau_{d}\right|}{\tau_{e}} \sin \varphi\right)+n_{d}(t) g(t-\hat{\tau}) \sin \left(\omega_{0} t+\hat{\psi}\right) \\
& \xi_{1}(t) \frac{d g(t-\hat{\tau})}{d \hat{\tau}} \cos \left(\omega_{0} t+\hat{\psi}\right)=\frac{1}{2} A g(t-\tau) \frac{d g(t-\hat{\tau})}{d \hat{\tau}} \cos \varphi+n_{d}(t) \frac{d g(t-\hat{\tau})}{d \hat{\tau}} \cos \left(\omega_{0} t+\hat{\psi}\right) .
\end{aligned}
$$

Besides, during time 0 the variable $\varphi$ and $\tau_{d}$ it should be noted that the constant time for PLL and DTS in operating electronics is typically greater than the duration of the information symbol.

Note that $F\left(\tau_{d}\right)$ is wider than the dynamical characteristics of the DTS DTS $F_{d y n}$, which is described by the expression

$$
F_{d y n}=1-\frac{\left|\tau_{d}\right|}{\tau_{e}} F\left(\tau_{d}\right),
$$

the limits of which correspond to point $\tau_{d}= \pm \tau_{e}$.

The dispersion of random the processes $n_{1}(t), n_{2}(t)$ end $n_{3}(t)$ is determined using the following formulas:

$$
\sigma_{n_{1}}^{2}=\frac{1}{4} N_{0} T_{0}, \quad \sigma_{n_{2}}^{2}=\frac{1}{4} N_{0} T_{0}, \quad \sigma_{n_{3}}^{2}=\frac{1}{2} N_{0} T_{0} \frac{1}{\tau_{e}^{2}}, \quad k_{n 1 n 2}=k_{n 1 n 3}=k_{n 2 n 3}=0,
$$

where $\sigma_{n_{1}}^{2}, \sigma_{n_{2}}^{2}, \sigma_{n_{3}}^{2}$ are respective to the dispersion of the random processes $n_{1}(t), n_{2}(t), n_{3}(t)$; $k_{n 1 n 2}, k_{n 1 n 3}, k_{n 2 n 3}$ are the functions of their mutual correlation.

The differential equations (1) fully describe the statistical dynamics of the tracking systems in a radio receiver for noise-like signals possessing inverse modulation with phase fluctuation and signal delay in an additive noise environment. For time intervals $t_{i} \lg \Delta \tau$, where $\Delta \tau>>\tau_{c}, \tau_{c}=T_{0}$ is the random processes correlation time $n_{1}(t), n_{2}(t), n_{3}(t)$, equation (1) describes a bidimensional Markov chain $\left(\varphi, \tau_{d}\right)$, the deviation coefficient and diffusion of which are determined by 
the following formulas

$$
\begin{gathered}
a\left(\lambda_{i}\right)=\frac{M\left[\lambda_{i}(t+\Delta \tau)-\lambda_{i}(t)\right] \mid\left(\lambda_{i} \lambda_{j}\right)}{\Delta \tau} \\
b\left(\lambda_{i} \lambda_{j}\right)=\frac{M\left[\lambda_{i}(t+\Delta \tau)-\lambda_{i}(t)\right]\left[\lambda_{j}(t+\Delta \tau)-\lambda_{j}(t)\right] \mid\left(\lambda_{i} \lambda_{j}\right)}{\Delta \tau}
\end{gathered}
$$

where $\lambda_{1}=\varphi, \lambda_{1}=\tau_{d}, \mathrm{i}=1,2, \mathrm{j}=1,2$. Using a technique for calculating the deviation coefficient and the diffusion of the Markov chain [7], and considering (3), we will get for equation (1):

$$
\begin{aligned}
& a(\varphi)=-\alpha \varphi-\Delta \varphi \sin 2 \varphi, \quad a\left(\tau_{d}\right)=-\beta \tau_{d}+\Delta \tau F\left(\tau_{d}\right), \\
& b\left(\tau_{e}, \tau_{e}\right)=\frac{2 \Delta^{2} \tau \delta}{\cos ^{2} \varphi\left(1-\frac{\tau_{d}}{\tau_{e}}\right)^{2}}\left[F^{2}\left(\tau_{d}\right)+2\left(1-\frac{\tau_{d}}{\tau_{e}}\right)^{2}+\frac{2 \delta}{T_{0} \cos ^{2} \varphi}\right]+\frac{1}{2} N \tau, \\
& b(\varphi, \varphi)=\frac{8 \Delta^{2} \varphi \delta}{\left(1-\frac{\tau_{d}}{\tau_{e}}\right)^{2}}\left[1+\frac{\delta}{T_{0}\left(1-\frac{\tau_{d}}{\tau_{e}}\right)^{2}}\right]+\frac{1}{2} N_{\varphi}, \\
& b\left(\varphi, \tau_{d}\right)=\frac{4 \sin \varphi \Delta \varphi \Delta \tau F\left(\tau_{d}\right) \delta}{\left(1-\frac{\tau_{d}}{\tau_{e}}\right)^{2} \cos \varphi},
\end{aligned}
$$

where $\delta=\frac{N_{0}}{A^{2}}$ is the ratio of the spectral noise density to the square of the signal amplitude.

The coefficients in equation (5) enable us to determine the probability and time characteristics for signal tracking failure in both DTS and PLL simultaneously; this is performed on the basis of the numerical solution for Pontryagin's second equation $[3,4,5]$, which relative to k-th moment of $t_{k}$ time of the first advance of the phase trajectory and delay of the work zone limits signal $|\varphi|=\frac{\pi}{2},\left|\tau_{d}\right|=\tau_{e}$ from the starting point $\left(\varphi_{0}, \tau_{d 0}\right) \in\left\{|\varphi| \leqslant \pi / 2,\left|\tau_{d}\right| \leqslant \tau_{e}\right\}$ will become

$$
\begin{gathered}
\left\{\frac{4 \Delta^{2} \varphi \delta}{\left(1-\frac{\left|\tau_{d_{0}}\right|}{\tau_{e}}\right)^{2}}\left[1+\frac{\delta}{T_{0}\left(1-\frac{\left|\tau_{d_{0}}\right|}{\tau_{e}}\right)^{2}}\right]+\frac{1}{4} N_{3}\right\} \frac{d^{2} t_{k}}{d \varphi_{0}^{2}}+ \\
+\left\{\frac{\Delta^{2} \tau \delta}{\cos ^{2} \varphi_{0}\left(1-\frac{\left|\tau_{d_{0}}\right|}{\tau_{e}}\right)^{2}}\left[F^{2}\left(\tau_{d_{0}}\right)+2\left(1-\frac{\left|\tau_{d_{0}}\right|}{\tau_{e}}\right)^{2}+\frac{2 \delta}{T_{0} \cos ^{2} \varphi_{0}}\right]+\frac{1}{4} N \tau\right\} \times \\
\times \frac{d^{2} t_{k}}{d \tau_{d_{0}}^{2}}+\frac{4 \sin \varphi_{0} \Delta \varphi \Delta \tau F\left(\tau_{p_{0}}\right) \delta}{\left(1-\frac{\left|\tau_{d_{0}}\right|}{\tau_{e}}\right)^{2} \cos \varphi_{0}} \cdot \frac{d^{2} t_{k}}{d \varphi_{0} d \tau_{d_{0}}}-\left(\alpha \varphi_{0}+\sin 2 \varphi_{0}\right) \frac{d t_{k}}{d \varphi_{0}}- \\
-\left[\beta \tau_{d_{0}}-\Delta \tau F\left(\tau_{d_{0}}\right)\right] \frac{d t}{d \tau_{d_{0}}}=-k t_{k-1},
\end{gathered}
$$

where $t_{0}=1, k=1,2, \ldots, t_{k}=0$ provided $|\varphi|=\pi / 2,\left|\tau_{p 0}\right|=\tau_{\mathrm{e}}$.

According to (6), the calculation of the probability and time characteristics of the signal tracking failure incorporates the following expressions that characterize the equivalent PLL and DTS bands

$$
f_{n} \varphi=\Delta \varphi+\alpha / 2 ; \quad f_{n} \tau=\frac{\Delta \tau}{2 \tau_{e}}+\frac{\beta}{2},
$$

where $f_{n} \varphi$ and $f_{n} \tau$ are the equivalent PLL and DTS bands. 
If we substitute in (5) the values $\Delta \varphi, \Delta \tau, \bar{k}_{11}, \bar{k}_{22}$ and considering the fact these expressions have been determined for the lesser values $\varphi$ and $\tau_{p}$, we will get

$$
\begin{gathered}
f_{n} \tau=\frac{1}{2} \beta \sqrt{1-\frac{\bar{\nu}_{222} N \tau}{2 \beta^{2}}} ; \quad f_{n} \varphi=\frac{1}{2} \alpha \sqrt{1-\frac{\bar{\nu}_{211} N \psi}{2 \alpha^{2}}} ; \\
\bar{\nu}_{211}=-\frac{A^{4} T_{0}}{N_{0}^{2}} ; \quad \bar{\nu}_{222}=-\frac{A^{4} T_{0}}{N_{0}^{2} \tau_{e}^{2}}
\end{gathered}
$$

In order to bring the discussed optimal PLL and DTS models in line with existing tracking systems, it is desirable during calculating the probability and time characteristics of tracking failures to fix their equivalent bands at a level, which provides maximum interference immunity of the radio receiver at given instabilities of the base generator.

An admissible error due to the instability of the base generator in equal to $0,1 \eta$, where $\eta$ $\mathrm{s}$ the width of the discriminate characteristics of the tracking system. From this condition, for the given instability of the base generator $\Delta f_{g}$ we shall obtain a minimal admissible value of the equivalent band

$$
f_{n}=\frac{\Delta f_{g} f_{g}}{0,1}
$$

where $f_{g}$ is the operating frequency of the base generator.

Thus, when calculating the probability and time characteristics of tracking failure (6), which are used to determine the effectiveness of the impact of noise on the receiver, the following procedure is used to acquire initial data:

1) the instabilities of the base generators, used in the device, are understood;

2) the equivalent bands PLL and DTS $\left(f_{g} \varphi f_{g} \tau\right)$ are determined using the equation (9);

3 ) in equation (6), the following is accepted

$$
\left.\Delta \varphi=\left(f_{n} \varphi-\frac{\alpha}{2}\right)\left(1-\frac{\tau_{e_{0}}}{\tau_{e}}\right)^{2} ; \Delta \tau=\left(2 \tau_{e} f_{n} \tau-\beta \tau_{e}\right)\left(1-\frac{\left|\tau_{e_{0}}\right|}{\tau_{e}}\right) \cos ^{2} \varphi\right) .
$$

From equation (7) with the ratio $\frac{N_{0}}{A^{2}} \leqslant 1$, which corresponds to the operation of a radio receiver during natural noise, we find the values $N_{\varphi}$ and $N_{\tau}$. To simplify the calculation, we can accept that $\alpha=f_{n} \varphi, \beta=f_{n} \tau$.

Solving elliptical differential equations (6), having substituted the numerical values of the parameters, which characterize the satellite signals, allows us to obtain the quantitative values of the average time and time dispersion to the moment of tracking failure in radio receivers of command and measuring systems in noise environments, having an intensity of $N_{0}$.

Since it has not been possible to solve this equation analytically [3, 8], the solution is sought for using numerical methods and the famous tridiagonal matrix algorithm [3]. In order to enable a high quality analysis of the influence of inverse modulation and cross coupling between PLL and DTS on the effectiveness of noise influence on the receiving device, the solution of the equation (6) is performed for the following variants:

- for PLL supposing that DTS has an ideal performance, i.e.

$$
\tau_{d 0}=0, \quad \frac{d t_{k}}{d \tau_{d_{0}}}=0, \quad \frac{d^{2} t_{k}}{d \tau_{d_{0}}^{2}}=0, \quad \frac{d^{2} t_{k}}{d \varphi_{0} d \tau_{d_{0}}}=0 ;
$$

- for DTS supposing that PLL has an ideal performance, i.e. 


$$
\varphi_{0}=0, \quad \frac{d t_{k}}{d \varphi_{0}}=0, \frac{d^{2} t_{k}}{d \varphi^{2}}=0, \frac{d^{2} t_{k}}{d \varphi_{0} d \tau_{d_{0}}}=0 ;
$$

- for joint performance of PLL and DTS.

When solving the equation, the values of the parameters of the radio signals are taken as: $f_{g \varphi}=1500 \mathrm{MHz} ; f_{g \tau}=10 \mathrm{MHz} ; \tau_{e}=0,1 \mu s$, where $f_{g \varphi}$ is the frequency of the DTS base generator; $f_{g \tau}$ is the frequency of the PLL base generator. The frequency instability of the base generator is accepted as equal to $10^{-9}$. The solution of the equation (6) was performed for two values of $T_{0}: 10^{-3} \mathrm{~s}$ and $2 \cdot 10^{-2} \mathrm{~s}$. The initial tracking errors for frequency and phase were supposed as equal to errors caused by the instability of base generators, i.e. $\varphi_{0}=0,1 \pi$, $\tau_{0}=0,01 \mu s$.

To get the results of the value, $N_{\tau}$ and $N_{\varphi}$, were determined for $d=10^{-4}$. The further decrease of d while finding the values of $N_{\varphi}$ and $N_{\tau}$ did not significantly affect the results if the calculation; this allows us obtain guarantied assessment during investigations in conditions of greater ambiguity for possible real values of $N_{\varphi}$ and $N_{\tau}$.

During calculations, we have determined that the value of the average time to tracking failure corresponds to the mean square value of time deviation to tracking failure.

\section{Conclusion}

Based on the conducted analysis, we can conclude that during the evaluation of the noise immunity of a radio receiver operating in the tracking mode in noise environments, it is enough to study the tracking failure in the PLL at real initial shifts of signal frequency and phase and the ideal operating of the DTS. Let us determine the threshold value of the noise to signal ratio th or a noise interference which matches the signal spectrum. Considering that actual interfering signal can be replaced by a fictive flat noise with a correlative function,

where $N_{0}=\frac{2 P_{n}}{\Delta f_{n}}, P_{n}$ is the noise level at output from the receiver.

$$
K_{t h}\left(t_{2}-t_{1}\right)=\frac{N_{0}}{2} \delta\left(t_{2}-t_{1}\right)
$$

In result we get $K_{t h}=\left(P_{n} / P_{s}\right)_{t h}=4 \delta_{n} / \Delta f_{n}$, where $P_{n} / P_{s}$ is the ratio between the noise level to the signal at output.

Considering that in real receivers more than half of the signal power is lost during processing [9], we shall get

$$
K_{t h}=2 \delta_{n} / \Delta f_{n} .
$$

The results of the calculations using the formula (10) and considering the calculated above threshold values of the ratio $\frac{N_{0}}{A^{2} T_{0}}$ are given in the Tab. 1 .

Table 1. Threshold values of the noise to signal ratio for noise

\begin{tabular}{|l|l|l|l|l|l|l|}
\hline$f_{n \varphi}, \mathrm{Hz}$ & 6 & 10 & 25 & 40 & 50 & 70 \\
\hline$K_{t h}, \mathrm{~dB}$ & 37 & 36 & 30 & 27 & 26 & 25 \\
\hline
\end{tabular}

The realizable values of the minimal width of the equivalent band of the PLL system for radio receiving devices of command and measuring systems are estimated at $20 \mathrm{~Hz}$. Consequently, the threshold ratio of noise to signal is respectively $42 \mathrm{~dB}$ and $32 \mathrm{~dB}$.

This study has been funded by the Ministry of Education and Science of the Russian Federation (Agreement 14.577.21.0220 dated 03.10.2016; unique project identifier: RFMEFI57716X0220). 


\title{
References
}

[1] H.B.Hassrizal, J.A.Rossiter, A survey of control strategies for spacecraft attitude and orientation, 2016 UKACC 11th International Conference on Control (CONTROL), 2016.

[2] Y.Ephraim, N.Merhav, Hidden Markov processes, IEEE Transactions on Information Theory, 48(2002), no. 6, 1518-1569.

[3] R.Rudnicki, M.Tyran-Kaminska, Markov processes, SpringerBriefs in Applied Sciences and Technology, Issue 9783319612935, 2017, 33-62.

[4] V.I.Tikhonov, M.A.Mironov, Markov Processes, Sov. Radio, Moscow, 1977 (in Russian).

[5] W.C.Lindsey Synchronization Systems in Communication and Control, Englewood Cliffs NJ, Prentice-Hall, 1972.

[6] J.J.M.Wang, R.D.Cideciyan, W.C.Lindsey, Phase and frequency transfer with automatic doppler and delay compensation, GLOBECOM'86, IEEE Global Telecommunications Conference: Communications Broadening Technology Horizons, Conference Record., Houston, TX, USA, 1986.

[7] L.M.Fink, The theory of transfer discrete messages, Sov. Radio, Moscow, 1970 (in Russian).

[8] A.A.Kovalchuk, Cycle skip in discrete phase-lock system., Scientific periodical of the Bauman $M S T U$, 10(2012) (in Russian).

[9] N.I.Andrusenko, T.N.Kachalina, Yu.G.Nikitenko, Comparative estimation of noise-stability characteristics in the presence of noise-like interference in specialized systems of information exchange, Radioelectronics and Communications Systems, 50(2007), no. 2, 81-86.

\section{Нелинейные методы статистического анализа динамики следящих систем радиоприемных устройств}

\author{
Владимир А. Миронов \\ Военный учебно-научный центр ВВС \\ Старых Большевиков, 54a, Воронеж, 394064 \\ Россия \\ Дмитрий Д. Дмитриев \\ Валерий Н. Тяпкин \\ Алексей Ю. Ершов \\ Военно-инженерный институт \\ Сибирский федеральный университет \\ Свободный, 79, Красноярск, 660041 \\ Россия
}

\begin{abstract}
В статье проведена оценка критериальных отношений (требуемых защитных отношений) сигнал/помеха, обеспечивающих функиионирование радиоприемного устройства в условиях дефицита помехозащищенности с вероятностъю не менее 0,9. Оценка проведена с исполъзованием теории марковских процессов на основе нелинейных методов анализа статистической динамики следящих систем и теории оптимальной фильтрачии информационных прочессов.
\end{abstract}

Ключевые слова: адаптивные радиоприемные устройства, помехоустойчивость, слежение, оптимальный алгоритм, синтез. 